\title{
FUSION IGNITION RESEARCH EXPERIMENT (FIRE)
}

\author{
Dale M. Meade \\ Princeton Plasma Physics Laboratory \\ P.O. Box 451 \\ Princeton, NJ 08543 \\ (609)-243-3301
}

\section{ABSTRACT.}

Experiments are needed to test and extend present understanding of confinement, macroscopic stability, alpha-driven instabilities, and particle/power exhaust in plasmas dominated by alpha heating. A design study of a Fusion Ignition Research Experiment (FIRE) is underway to assess near term opportunities for producing and studying fusion dominated plasmas in the laboratory. The emphasis is on understanding the behavior of fusion plasmas dominated by alpha heating $(Q \geq 5)$ that are sustained for a duration comparable to the characteristic plasma time scales $\left(\geq 20 \tau_{\mathrm{E}}\right.$ and $1.5 \tau_{\text {skin }}$, where $\tau_{\text {skin }}$ is the time for the plasma current profile to redistribute at fixed current). These requirements can be satisfied with $\mathrm{BeCu} / \mathrm{OFHC}$ toroidal field coils and OFHC poloidal coils that are pre-cooled to $77^{\circ} \mathrm{K}$ prior to the pulse. The plasma facing components will have tungsten divertor plates and Be first wall tiles. No graphite is allowed inside the vacuum vessel due to tritium retention issues. The mission of FIRE is to attain, explore, understand and optimize alpha-dominated plasmas to provide knowledge for the design of attractive magnetic fusion energy systems. The programmatic strategy is to access the alphaheating-dominated regime with confidence using the present advanced tokamak data base (e.g., Elmy-H-mode, $\leq 0.75$ Greenwald density) while maintaining the flexibility for accessing and exploring other advanced tokamak modes (e. g., reversed shear, pellet enhanced performance) at lower magnetic fields and fusion power for longer durations in later stages of the experimental program. A major constraint is to develop a design concept that could meet these physics objectives with a construction cost in the range of $\$ 1 B$.

\section{INTRODUCTION}

Burning plasma physics has been accepted as the primary objective for a next step for both inertial and magnetic fusion. Billion dollar scale facilities, NIF in the U.S. and LMJ in Europe, are now under construction to study the physics of inertially confined burning plasmas. Numerous reviews of the U.S. fusion program have recommended the development of a plan for a burning plasma physics research. In 1999, the Secretary of Energy's Advisory Board (SEAB) recommended that if Japan and Europe do not proceed with ITER, "the U.S. should pursue a less ambitious machine that will allow the exploration of the relevant science at a lower cost." "In any event the preliminary planning for such a machine should proceed now so as to allow prompt pursuit of this option." FIRE responds to this recommendation, and is envisioned as an extension of the existing advanced tokamak program leading to an attractive magnetic fusion reactor. ${ }^{1}$

\section{FUSION SCIENCE OBJECTIVES FOR FIRE}

The theme of FIRE is to provide a stepping stone between today's experiment's and physics 
understanding and an attractive fusion reactor. Successful implementation of FIRE and a long pulse advanced tokamak experiment such as the proposed JT-60 superconducting upgrade would provide much of the fusion plasma science basis needed for the design of an advanced fusion engineering test facility.

The fusion science objectives of FIRE are :

- Explore and understand the physics of alphadominated fusion plasmas

- energy and particle confinement (extend confinement predictability)

- macroscopic stability ( $\beta$-limit, neoclassical tearing modes, wall stabilization)

- Wave-particle interactions (fast alpha driven effects)

- Plasma boundary (density limit, power and particle flow)

- Strong coupling of preceding issues due to self-heating (self-organization)

- Test techniques to control and optimize alphadominated plasmas

- Sustain alpha-dominated plasmas

- High-power density exhaust of plasma particles and energy

- profile evolution induced by alpha-heating impacts on:

macro-stability transport barriers energetic particle stability

- Explore and understand some advanced operating modes and configurations that have the potential to lead to attractive fusion applications.

In short, we must Burn to Learn. The implementation of a burning plasma experiment will also drive several critical areas for plasma technology such as plasma facing components, high speed pellet launchers, high power-density ICRF heating and low inventory tritium systems.

\section{PLASMA PARAMETERS NEEDED FOR A BURNING PLASMA EXPERIMENT}

The objective of exploring the physics of a fusion plasma will require that dimensionless plasma variables $\left(\rho^{*}=\rho / a, v^{*}\right.$ and $\left.\beta\right)$ approach those in a fusion plasma. Kadomtsev ${ }^{2}$ has shown that the similarity parameter for the core of magnetic fusion plasmas is $\mathrm{BR}^{5 / 4}$, that is plasmas with the same $B^{5 / 4}$ can have the same $\rho^{*}=r / a$, $v^{*}$ and $\beta$, and hence will have the same plasma phenomena. A similarity parameter for edge physics has not been identified. Therefore, it is important for the edge plasma in a burning plasma experiment to be similar to that of a fusion reactor in dimensional terms. Alpha physics effects are quantified by the fraction of alpha heating, and the relative energy density of energetic alpha particles. A very important requirement is the duration of the burning plasma phase expressed in terms of the important plasma time constants, $\tau_{\mathrm{E}}$ the energy confinement time, $\tau_{\mathrm{He}}$ the alpha ash confinement time and $\tau_{\text {skin }}$ the time for plasma current perturbations to evolve. These dimensionless parameters are compared in Table I for JET/TFTR D-T Experiments, those anticipated

Table I. Dimensionless Parameters

\begin{tabular}{|l|c|c|l|l|l|}
\hline & Core & Alphas & \multicolumn{3}{|c|}{ Duration } \\
\hline & $\mathrm{BR}^{5 / 4}$ & $\mathrm{P}_{\alpha} \mathrm{P}_{\text {heat }}$ & $\tau / \tau_{\mathrm{E}}$ & $\tau / \tau_{\mathrm{He}}$ & $\tau / \tau_{\text {skin }}$ \\
\hline ARIES-AT & 1 & 0.9 & $>10$ & $>10$ & $>10$ \\
\hline FIRE Goal & 0.6 & $>0.5$ & $>10$ & $\sim 6$ & 1.5 \\
\hline TFTR/JET & 0.3 & 0.04 & $\sim 10$ & $\sim 2$ & $\sim 0.2$ \\
\hline
\end{tabular}

for ARIES $^{3}$ and the minimum goal for FIRE. FIRE's minimum goals will be more than half way to fusion plasma conditions and will have a duration sufficient to study the plasma response to alpha-dominated heating. 
The configuration chosen for FIRE is similar to that of ARIES-RS ${ }^{3}$, namely a highly shaped plasma, with double-null divertor and aspect ratio $\approx 4$. The FIRE design activities have focused on the physics and engineering evaluation of a compact, high-field tokamak with the parameters shown in Table II.

TABLE II. DESIGN GOALS FOR FIRE

\begin{tabular}{|l|c|}
\hline $\mathrm{R}(\mathrm{m}), \mathrm{a}(\mathrm{m})$ & $2.0,0.525$ \\
\hline $\mathrm{K}_{95}, \delta_{95}$ & $\approx 1.8, \approx 0.4$ \\
\hline $\mathrm{q}_{95}$ & $>3$ \\
\hline $\mathrm{B}_{\mathrm{t}}\left(\mathrm{R}_{\mathrm{o}}\right)(\mathrm{T})$ & $10(12)^{*}$ \\
\hline $\mathrm{Wmag} \mathrm{TF}(\mathrm{GJ})$ & 3.7 \\
\hline $\mathrm{I}_{\mathrm{p}}(\mathrm{MA})$ & $6.44(7.7)^{*}$ \\
\hline flattop time $(\mathrm{s})$ & $\sim 20(12)^{*}$ \\
\hline alpha heating fraction & $>0.5$ \\
\hline$\tau_{\mathrm{E}}, \tau_{\text {skin }}(\mathrm{s})$ & $\sim 0.6, \sim 13$ \\
\hline $\mathrm{Z}_{\text {eff }}\left(3 \%\right.$ Be + He $\left.\left(5 \tau_{\mathrm{E}}\right)\right)$ & 1.4 \\
\hline Fusion Power $(\mathrm{MW})$ & $100-200$ \\
\hline ICRF Power $(\mathrm{MW})$ & 30 \\
\hline Tokamak Cost $(\$ \mathrm{~B})$ & $\sim 0.3$ \\
\hline Project Cost $(\$ \mathrm{~B})$ & $\sim 1$ \\
\hline
\end{tabular}

* Higher Field Mode

The key "advanced tokamak" features are: strong plasma shaping, double null poloidal divertors, low toroidal field ripple $(<0.34 \%)$, internal control coils and space for the future addition of wall stabilization capabilities and additional auxiliary systems for plasma profile control. The magnets and structure are also capable of operation at $\mathrm{B}_{\mathrm{t}}\left(\mathrm{R}_{\mathrm{o}}\right)=12 \mathrm{~T}$ and $\mathrm{I}_{\mathrm{p}}=7.7$ MA with a flat top time of $12 \mathrm{~s}$ at $200 \mathrm{MW}$ of fusion power. Recently, an improved physics performance design point, FIRE*, has been identified with slightly lower aspect ratio (3.6 vs. $3.81)$, slightly increased triangularity ( 0.5 vs $0.4), R_{o}=2 \mathrm{~m}, \mathrm{a}=0.565 \mathrm{~m}, \mathrm{~B}_{\mathrm{t}}\left(\mathrm{R}_{\mathrm{o}}\right)=10 \mathrm{~T}$, and $\mathrm{I}_{\mathrm{p}}=$ 7.7 MA with a flat top time $\sim 15$ to 20 s at 125 MW of fusion power. The engineering feasibility of FIRE* is being evaluated.

\section{PHYSICS PROJECTIONS}

The physics design guidelines for FIRE are similar to those developed for ITER-FEAT ${ }^{4}$ from analysis of the H-Mode confinement database DB03v5(9) $;^{5}$

- Confinement is assumed to scale ${ }^{6}$ as ITER98 $(\mathrm{y}, 2)$ with an $\mathrm{H}$-factor determined by matching JET H-Mode data for FIRE-like conditions

- Operating density from $0.3<\mathrm{n} / \mathrm{n}_{\mathrm{GW}}<0.85$ where $\mathrm{n}_{\mathrm{GW}}$ is the Greenwald density

- $\mathrm{H}$-mode power threshold is given by $\mathrm{P}_{\text {th }}=2.84 \mathrm{n}_{20}{ }^{0.58} \mathrm{~B}^{0.82} \mathrm{Ra}^{0.81} \mathrm{M}^{-1}$

- $\beta_{\mathrm{N}}=\beta(\%) /\left(\mathrm{I}_{\mathrm{p}} / \mathrm{aB}\right)<2.5$

- Helium ash confinement $\tau_{\mathrm{He}}{ }^{*}=5 \tau_{\mathrm{E}}$, with $3 \% \mathrm{Be}$ impurities in the plasma core.

The operating regime for FIRE is well matched to the existing $\mathrm{H}$-mode database from $0.3<\mathrm{n} / \mathrm{n}_{\mathrm{GW}}<1.0$ as shown in Fig. 1. There is

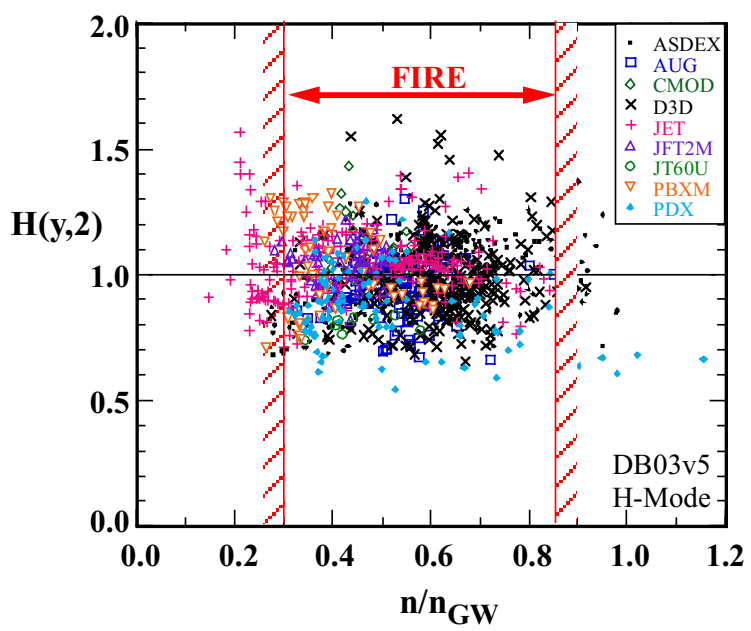

Fig. 1. H-Mode Database and FIRE

considerable scatter in the $\mathrm{H}$ factor from 0.7 to 1.5 for these cases. This is not random scatter since a given point can be reproduced if the identical conditions are repeated. The performance of FIRE was projected by selecting JET data ${ }^{7}$ from DB03v5(9) with parameters similar to FIRE, namely $\beta_{\mathrm{N}} \geq 1.7, \mathrm{Z}_{\text {eff }}<2.0, \kappa>$ 1.7 and $2.7<\mathrm{q}_{95}<3.5$. The average density profile peaking, $\mathrm{n}(0) /<\mathrm{n}>_{\mathrm{V}}$ for these FIRE-like JET points was found to be 1.2. Modeling of standard pellet injection scenarios indicates that peaking factors of 1.2 are feasible with high speed vertical launchers. Guided high field side pellet launch will be incorporated with the goal of providing additional density profile peaking up to 1.5 . The impurity level for these analyses 
was chosen to be $3 \% \mathrm{Be}$, where $\mathrm{Be}$ is the plasma facing material of the first wall, giving a $Z_{\text {eff }}$ $(\mathrm{imp})=1.36$. This assumption is consistent with the trend toward lower $Z_{\text {eff }}$ as the density is increased in Alcator C-Mod. ${ }^{8}$ The tungsten concentration in the plasma core must be below $\sim 10^{-5}$ to avoid significant radiation loss. It is assumed that tungsten from the divertor plate and any other impurities present in the divertor do not migrate to the plasma core. The use of tungsten coated tiles in the baffle region of the divertor of ASDEX Upgrade is encouraging with regard to maintaining acceptably low levels of tungsten in the plasma core. ${ }^{9}$

A 0-D power balance code was used to calculate the $\mathrm{H}$ factor required to achieve a given Q-value as shown in Fig. 2. The curves represent different cases with the density profile peaking of $n(0) /<n>_{V}=1.2$ or 1.5 with the design guidelines given above. Several FIRE cases were considered: Baseline configuration with 10 $\mathrm{T}$ and 6.44 MA, Baseline configuration at $12 \mathrm{~T}$ and $7.7 \mathrm{MA}$, and an improved geometry case (FIRE*) with $10 \mathrm{~T}$ and $7.7 \mathrm{MA}$. The shaded region indicates the confinement $\mathrm{H}(\mathrm{y}, 2)$ factors for elmy H-modes obtained on JET for conditions similar to those anticipated on FIRE, namely $\beta_{\mathrm{N}}>1.7,2.7<\mathrm{q}_{95}<3.5, \kappa>1.7$ and $Z_{\text {eff }}$ $<2$. This illustrates the well known sensitivity

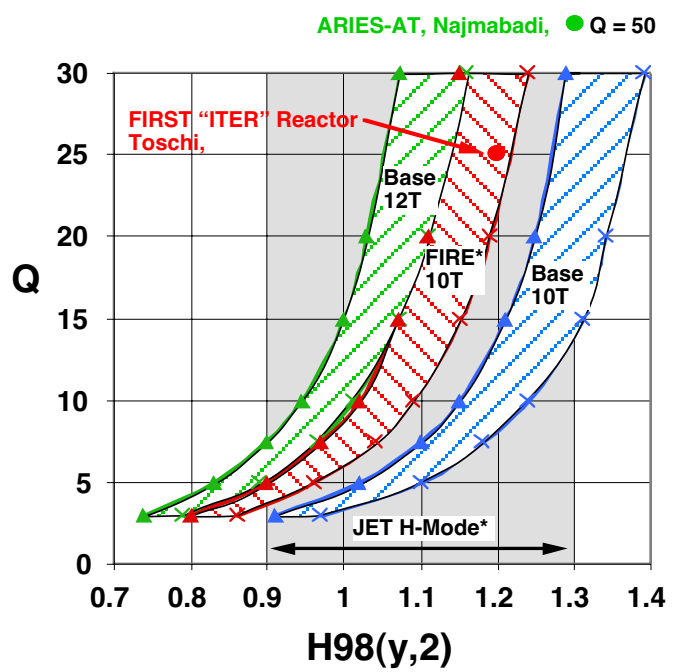

Fig. 2 Fire projections for three cases. Left edge of crosshatched band has density peaking of 1.5 and the right edge has 1.2. the Q value to confinement and to density profile peaking. Also shown are the H-factors (1.3 and 1.2) assumed for the U.S. reactor studies ${ }^{3}$ and for a first European reactor ${ }^{10}$ based on ITER. If FIRE can achieve similar $\mathrm{H}$-factors, then FIRE would also be able to access high $\mathrm{Q}$ regimes. and study regimes leading to attractive tokamak reactors.

Another important issue is the operating density range for a burning plasma experiment. The $\mathrm{H}$-factor required to achieve a given $\mathrm{Q}$ is plotted versus density normalized to the Greenwald density in Fig. 3a. The required Hfactor increases at high density where the plasma temperature decreases thereby reducing fusion reactivity. At low density, the required $\mathrm{H}$-factor

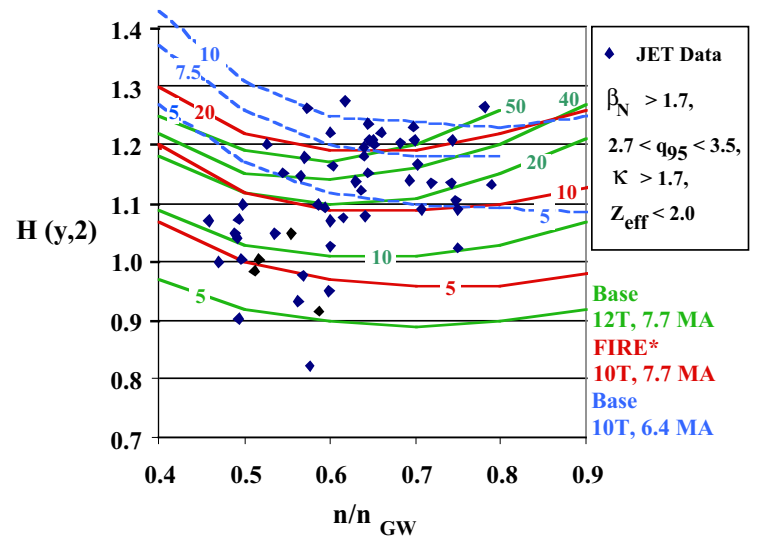

Fig. 3a. FIRE Projections with Q labels.

increases because $\tau_{\mathrm{E}}$ must increase to maintain $n \tau_{\mathrm{E}}$ approximately constant. Also shown are the FIRE-like JET H-modes points with $\beta_{\mathrm{N}}>1.7$, $2.7<\mathrm{q}_{95}<3.5, \kappa>1.7$ and $\mathrm{Z}_{\mathrm{eff}}<2$. The JET Hmode data have an average $<\mathrm{H}>\approx 1.1$ with an average density profile peaking of 1.2 .

The uncertainty in the projections of FIRE performance can be expressed as a fraction of the FIRE-like JET data points that would achieve a specific alpha-heating fraction $\mathrm{f}_{\alpha}=\mathrm{P}_{\text {alpha }} / \mathrm{P}_{\text {heat }}=$ $\mathrm{Q} /(\mathrm{Q}+5)$ as shown in Fig. 3b. The baseline configuration is projected to achieve the FIRE Mission requirement of $f_{\alpha} \geq 0.5(Q>5)$ for $53 \%$ of the FIRE-like JET points and would achieve 


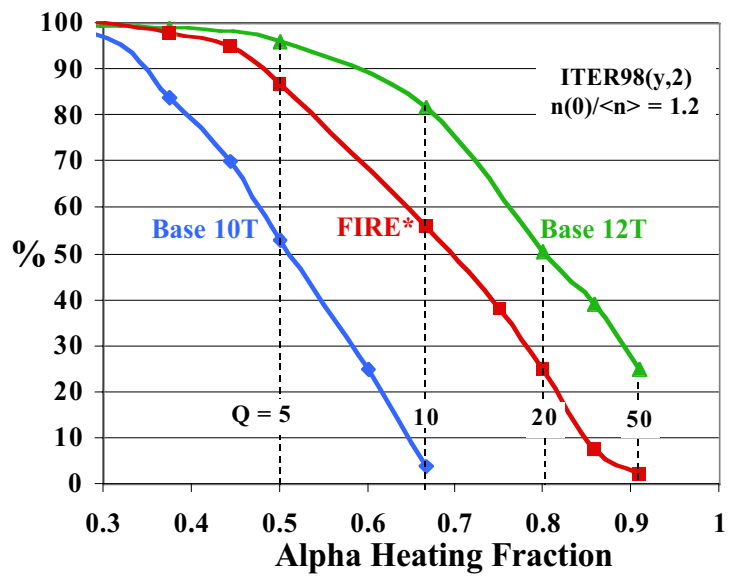

Fig. 3b. Per Cent of projected points achieving a specific alpha heating fraction.

$\mathrm{f}_{\alpha}>0.67(\mathrm{Q} \geq 10)$ for $4 \%$ of the data set. Operation of FIRE at 12T/7.7 MA or FIRE* the candidate design point (7.7 MA) have significantly higher performance with $>90 \%$ of the FIRE-like JET data projected to achieve Q $\geq 5,>50 \%$ projected to achieve $\mathrm{Q} \geq 10$ and $>25 \%$ projected to achieve $\mathrm{Q} \geq 20$.

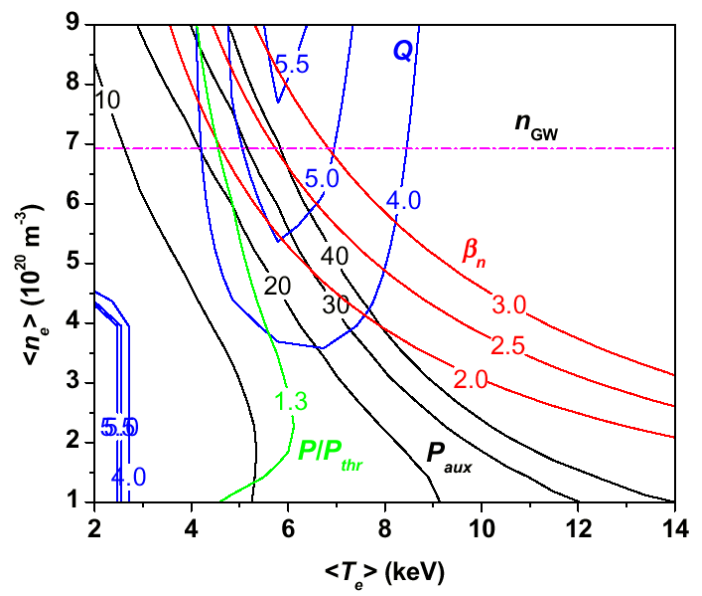

Fig. 4a Operating Space for FIRE (10T/6.44MA)

The operating space for FIRE can also be described using the PopCon plot as shown in Fig. 4 for cases similar to those described above with $\mathrm{H} 98(\mathrm{y}, 2)=1.1$ and $\mathrm{n}(0) /<\mathrm{n}>_{\mathrm{V}}=1.2$. The transported power exceeds the H-mode threshold by a factor of at least 1.3 to the right of the $\mathrm{P} / \mathrm{P}_{\text {thr }}$ $=1.3$ curve. The baseline FIRE (10T) attains alpha-dominated conditions but only over a

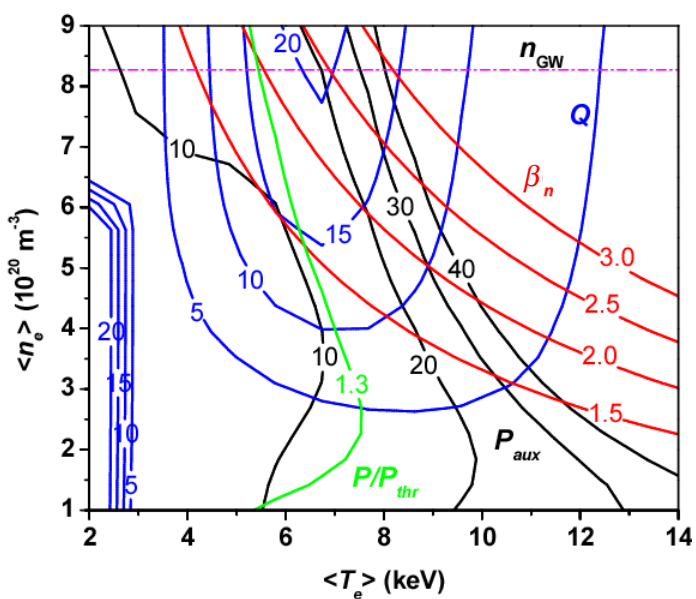

Fig. 4b. Operating Space for FIRE (12T/7.7MA)

limited range. The operating range for FIRE* and FIRE (12T) are similar, and achieve alphadominated conditions, $\mathrm{Q}>5$, over a large range and $\mathrm{Q}>10$ over a significant range.

A Tokamak Simulation Code (TSC) simulation of this regime with $\mathrm{H}(\mathrm{y}, 2)=1.03$ and $\mathrm{n}(0) /<\mathrm{n}>_{\mathrm{V}}=1.2$ indicates that FIRE can access the H-Mode and sustain alpha-dominated plasmas for $\approx 30 \tau_{\mathrm{E}},>6 \tau_{\mathrm{He}}$ and $\sim 1.5 \tau_{\text {skin }}$ as shown in Fig. 5. In addition, sufficient time is provided for plasma startup and a controlled shutdown to avoid plasma disruptions. The burn phase can study plasma profile evolution, alpha ash accumulation and techniques for burn control and begin studies of plasma current evolution due to alpha heating.

A longer term goal of FIRE is to explore advanced tokamak regimes using pellet injection

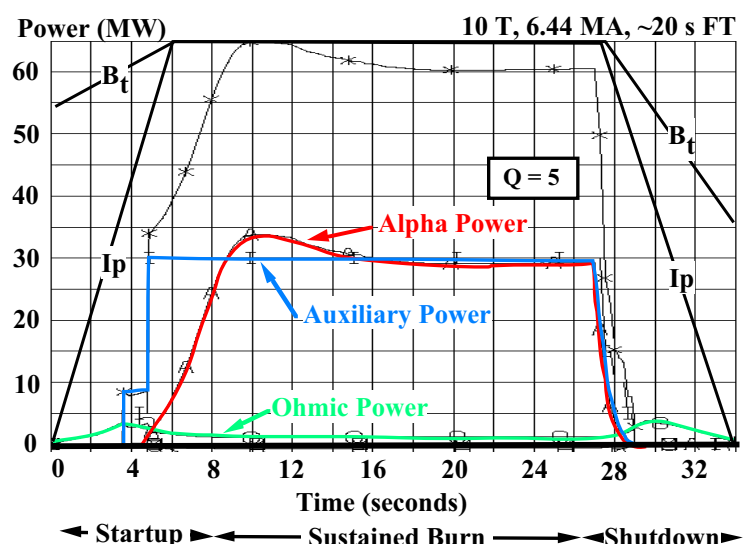

Fig. 5. Evolution of a fusion-dominated plasma. 
and current ramps to create reversed shear plasmas (e.g., PEP modes) for a duration of 1 to 3 current redistribution times. ${ }^{11}$ This AT capability is expected to produce modestly enhanced confinement and beta as observed in present large tokamak experiments, and would provide a continuous transition from $\mathrm{H}$-mode operation to advanced tokamak operation. An important feature of the FIRE cryogenic copper alloy magnets is that the pulse length increases rapidly as the field is reduced with flattops of $\sim 40 \mathrm{~s}$ at 8 $\mathrm{T}$ and $\sim 90 \mathrm{~s}$ at $6 \mathrm{~T}$. If confinement and $\beta$ are increased by 20 and $40 \%$ respectively, then the fields could be reduced by $20 \%$ and FIRE would have the capability to explore fusion-dominated plasmas for $40 \mathrm{~s}\left(\sim 3 \tau_{\text {skin }}\right)$. Physics scenarios and engineering solutions for power handling are not yet developed for the longer pulse $(\sim 40 \mathrm{~s})$ scenarios.

\section{TECHNOLOGY CONSIDERATIONS}

The engineering aspects of FIRE have been described elsewhere ${ }^{12-13}$, and in other papers at this conference, ${ }^{14}$ and are only summarized here. The baseline magnetic fields and pulse lengths can be provided with $\mathrm{BeCu} / \mathrm{OFHC}$ toroidal field (TF) coils and OFHC poloidal field (PF) coils that are pre-cooled to $77^{\circ} \mathrm{K}$ prior to the pulse and allowed to warm up to $373{ }^{\circ} \mathrm{K}$ at the end of the pulse [7]. The cross-section of FIRE is shown schematically in Fig. 6. The 16 TF coil system is wedged with a compression ring to resist de-wedging at the top and bottom of the inner TF leg. Shielding is added between the walls of a double wall vacuum vessel to reduce nuclear heating of the coils, limit insulation dose and allow hands-on maintenance outside the envelope of the TF coils within a few hours after a full power D-T shot. Large $(1.3 \mathrm{~m}$ by $0.7 \mathrm{~m})$ midplane ports provide access for heating, diagnostics and remote manipulators, while 32 angled ports provide access to the divertor regions for utilities and diagnostics. FIRE is being designed mechanically to accommodate 3,000 full field, full power pulses and 30,000 pulses at $2 / 3$ field. The repetition time at full field and full pulse length will be $<3 \mathrm{hr}$, with shorter times at reduced field or pulse length. The fusion energy production of $5 \mathrm{TJ}$ (similar to $\mathrm{BPX}$ ) produces a lifetime neutron dose to the TF insulating material at the inboard midplane of $\approx$ $1.5 \times 10^{10}$ Rads which is consistent with the polyimide insulation being considered.

The power densities on the divertor plates are $\sim 6 \mathrm{MWm}^{-2}$ for detached operation and $\sim 25$ $\mathrm{MWm}^{-2}$ for attached operation [8]. Carbon is not allowed in the vessel due to tritium inventory build-up by co-deposition. The divertor plasmafacing components are tungsten "brush" targets mounted on copper backing plates, similar to a concept developed by the ITER R\&D activity. The outer divertor plates and baffle are watercooled, while the inner divertor targets and first wall are cooled by mechanical attachment to water-cooled copper plates inside the vacuum vessel. The first wall is comprised of Be plasmasprayed onto copper tiles. The high neutron wall loading $\left(3 \mathrm{MWm}^{-2}\right)$ at full fusion power of 200 MW contributes significantly to the first wall and vacuum vessel heating. The water-cooled copper plates inside the vessel alleviate excess heating of the stainless steel vessel due to neutrons. Sixteen cryo-pumps - closely coupled to the divertor chambers, but behind sufficient neutron shielding - provide pumping $(\geq 100 \mathrm{~Pa}$ $\mathrm{m}^{3} / \mathrm{s}$ ) for D-T and $\mathrm{He}$ ash during the pulse. Pellet injection scenarios will help minimize tritium throughput. The in-device tritium inventory will be determined primarily by the cycle time of the divertor cryo-pumps, and can range from $<2 \mathrm{~g}$ for regeneration overnight to $\sim 20 \mathrm{~g}$ for monthly regeneration.

\section{SUMMARY AND FUTURE ACTIVITIES}

The present engineering design exceeds the original design goals. The confinement analysis based on ITER98(y,2) scaling indicates the importance of increasing the plasma current in FIRE to $\sim 8 \mathrm{MA}$. This could be accomplished in the baseline design operated at $12 \mathrm{~T}$ for $12 \mathrm{~s}$ flattop. An important requirement is to maintain a $20 \mathrm{~s}\left(1.5 \tau_{\mathrm{E}}\right)$ flattop as the current is increased. The design refinement of increasing the plasma 
shaping factor as in FIRE* is being evaluated along with the possibility of using only high conductivity (OFHC) copper in the TF coil. This lower strength material would require the addition of TF coil bucking on the central solenoid coils near the midplane. Initial results suggest that $11.5 \mathrm{~T}$ could be produced with a flattop of $\approx 25 \mathrm{~s}$ using about $1 / 2$ the electrical power required by the baseline $\mathrm{BeCu} \mathrm{TF}$ coil. The limitation on burn time for both $\mathrm{BeCu}$ and OFHC TF coil designs is the power handling capability of plasma facing components and the vacuum vessel.

A number of other important physics and engineering issues remain to be addressed during the remainder of the design study including generic issues such as: mitigation and avoidance of disruptions and vertical displacement events, effects of neoclassical tearing modes, detached divertor operation with good confinement, and divertor/edge plasma modeling under high power conditions.

\section{ACKNOWLEDGEMENTS}

The FIRE design study is a U.S. national activity managed through the Virtual Laboratory for Technology and is supported by USDOE Contract \#DE-AC02-76-CH03073.

\section{REFERENCES}

1. D. M. Meade; "Fusion Ignition Research Experiment", Comments on Plasma Phys. Controlled Fusion, Vol 2(2), pp. 81, 2000.

2. B. B. Kadomtsev, Sov. J. Plasma Phys. 1, 295 (1975)

3. R. Aymar et. al., Nuc. Fus. 30, No. 12, p 2208 (1999)

4. F. Najmabadi, et al., Fusion Eng. Design 38 (1997) 1-218

5. M. Shimada, et al., "Physics Basis of ITERFEAT” ITERP/05, Fusion Energy 2000
(Proc. $18^{\text {th }}$ Int. Conf. Sorrento, 2000), IAEA, Vienna (2000)

6. O. J. Kardun, et al., "Next Step Tokamak Physics: Confinement Oriented Global Database Analysis" ITERP/04, Fusion Energy 2000 (Proc. 18 ${ }^{\text {th }}$ Int. Conf. Sorrento, 2000), IAEA, Vienna (2000)

7. L. D. Horton, et al., "Pellet Fueling and ELMy H-mode Physics at JET" EX2/1, Fusion Energy 2000 (Proc. $18^{\text {th }}$ Int. Conf. Sorrento, 2000), IAEA, Vienna (2000)

8. M. Greenwald, Phys. Plasmas 2 (6), (1995) 2308-2313

9. R. Neu, et al., "Plasma Operation with Tungsten Tiles in ASDEX Upgrade, $14^{\text {th }}$ Plasma Surface Interactions in Controlled Fusion Devices Conference, Rosenheim (2000)

10. R. Toschi, "How Far is a Fusion Power Reactor from an Experimental Reactor", $21^{\text {st }}$ Symp. on Fusion Tech., Madrid (2000)

11. W. Houlberg, "Burning Plasma Physics Issues Illustrated by Simulations of FIRE". $14^{\text {th }}$ ANS Topical Meeting on Fusion Technology (2000)

12. Thome, R. J. et al., "Engineering Features of the Fusion Ignition Research Experiment (FIRE)", $21^{\text {st }}$ Symp. on Fusion Tech., Madrid (2000)

13. Ulrickson, M. A. et al., "Physics Basis for the Fusion Ignition Experiment (FIRE) Plasma Facing Components", $21^{\text {st }}$ Symp. on Fusion Tech., Madrid (2000)

14. Engineering Status of the Fusion Ignition Research Experiment (FIRE), R.J. Thome, P.J. Heitzenroeder, $14^{\text {th }}$ ANS Topical Meeting on Fusion Technology (2000) 